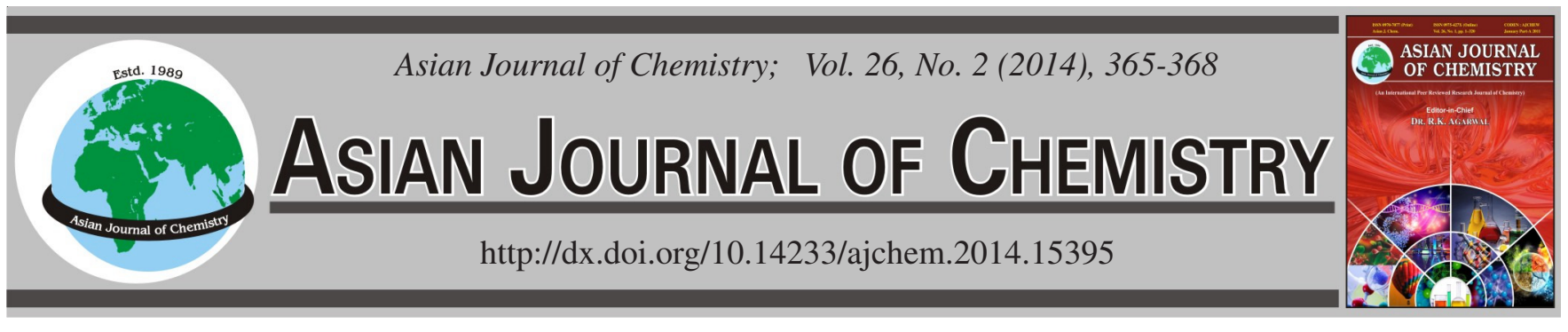

\title{
Phytochemical Investigation of Bioactive Extract from Endemic Medicinal Plant Limoniastrum feei (Girard) Batt (Plumbaginaceae)
}

\author{
Smahia Rahmani ${ }^{1}$, NASSER Belboukhari ${ }^{1, *}$ and AbDelkrim Cheriti $^{2}$
}

${ }^{1}$ Bioactive Molecules and Chiral separation Laboratory, University of Bechar, Béchar 08000, Algeria

${ }^{2}$ Phytochemistry and Organic Synthesis Laboratory, University of Bechar, Béchar 08000, Algeria

*Corresponding author: E-mail: belboukhari.nasser@yahoo.com

\begin{abstract}
The phytochemical investigation of bioactive extract of aerial part of Limoniastrum feei led to isolation of eleven polyphenol compounds, with two major constituents obtained from the bioactive extract of stems part, identified as glucosylated flavonoids: 3-O-(glucoside-6"malonyl, 2"-ramnosyl)-7,4',5'-trimethoxy-3-hydroxy flavonol and tetraacetate 7,8-dihydroxy-4'-methoxyisoflavone 8-O- $\beta$-glucopyranoside. The compounds were isolated for the first time from the plant extracts by column chromatography and prep-HPLC and structural elucidation was carried out on the basis of their spectral analysis.
\end{abstract}

Keywords: Limoniastrum feei, Plumbaginaceae, Flavones, Isoflavone, LC-UV, TLC.

\section{INTRODUCTION}

Limoniastrum feei (plumbaginaceae) is a herbaceous medicinal plant endemic to Algeria and Morocco Sahara. The aerial parts of this plant were used in Sahara folk medicine for treating gastrointestinal tract aliments, fever, iceterus and various diseases. The plant is native to the southeast of Algeria (Saoura, region of Bechar), Northern Africa ${ }^{1-3}$.

According to our previous results, biological activity of several extracts of aerial parts (leaf, stem and twig) of L. feei against fungi and bacteria was evaluated by disc diffusion method. Now we are interested in isolating the natural compounds responsible of this biological activities ${ }^{4-6}$.

\section{EXPERIMENTAL}

The IR spectra $\left(v_{\max }\right)$ were determined on a AVATAR 320 FT-IR spectrophotometer. 1D and 2D NMR spectra were obtained on a Bruker Avance DRX 300 FT spectrometer operating at $300 \mathrm{MHz}$ for ${ }^{1} \mathrm{H}$ NMR and $75 \mathrm{MHz}$ for ${ }^{13} \mathrm{C}$ NMR. The LC system consisted of a liquid chromatography (Si-gel-230400 mesh, MerK- were used for column chromatography) operating at room temperature with a flow rate of $1 \mathrm{~mL} \mathrm{~min}^{-1}$ and the UNICAM UV 300 spectrophotometer detector operating at wavelength of $210 \mathrm{~nm}$.

Plant material: The whole plants of Limoniastrum feei were collected from Kenadza (region of Bechar) Algeria. The botanical identification and voucher specimen were deposited in the herbarium of the Phytochemical \& Organic Synthesis
Laboratory. The assigned specimen number was (CA99/14) ${ }^{3,6}$. The leaves, stems and twigs were separated, dried and ground into powder using a grinder.

Extraction and fractionation: Dried and ground stems, twigs and leaves of Limoniastrum feei (350 g) were extracted with acetone-water $(400 \mathrm{~mL}, 75 \%)$. The extract was concentrated in water and partitioned with hexan, diethyle ether, ethyle acetate and $n$-butanol $(50 \mathrm{~mL} \text { in } 3 \text { steps })^{7}$. To purify and identify the constituents of three fractions; ethyle ether, ethyle acetate and butanol, separation by liquid chromatography coupled with UV detector (LC-UV) was developed in our laboratory ${ }^{8,9}$.

\section{RESULTS AND DISCUSSION}

The protocol used for isolation of these natural substances of the plant is made in two stages: In the first one makes a solid-liquid extraction with a solvent appropriate to the ground plant organ. The second step is a liquid-liquid fractionation of the crude extract of plant organs in three solvents of different polarity ${ }^{10}$.

The yield mass fractions are related to the polarity of the solvent, it is noted that the butanol extract is the most important (0.81-1.51\%). The TLC analysis, was performed; on TLC plate of silica gel eluting by two systems of different polarity with: acetone/toluene/formic acid $(6 / 8 / 1)^{4,11}$. The results of this study and analytical extraction are summarized Table- 1 . Table-1 shows the presence of several compounds in the ether fraction and ethyl acetate, by visualizing a spot against in the butanol 
fraction at $R_{\mathrm{f}}=0.85$, with absorbance at $254 \mathrm{~nm}$ color purple but this spot we had not seen, when eluting with E1.

TLC analysis of stem extract shows 4 components in all three fractions, 3 components in both ether fraction and ethyl acetate and were sometimes separated a compound in the butanol extract has an $R_{f}=0.57$. We reverse the order of fractionation starts with the most polar solvent (Table-1).

TLC analysis also shows the presence of 4 components in all three fractions, two in butnol extract (first extract). It has happened has separated the same compound $\left(R_{\mathrm{f}}=0.57\right)$ in the ether fraction and another is in the extract of ethyl acetate $R_{f}=$ 0.17 .

The analytical results indicate the presence of 5 total compounds ( 5 spots), including the compound at $R_{\mathrm{f}}=0.50$ appears in all three fractions. It is noted that the two fractions ether and ethyl acetate, contain almost the same constituents. Compared against a sample of stem, we note the presence of the compound at $\mathrm{R}_{\mathrm{f}}=0.66$ in both fraction (ether and ethyl acetate) of the two parts of the plant.

The phytochemical screening of three parts of Limoniastrum feei on tannins and glycosylated flavonoid, show presence of flavonoid in ethyl acetate and butanol fraction in considerable quantities and total absence in ethyl ether, but the tannin derivative are present in all parts.

It is also observed that the extracts of ethyl acetate and butanol are the richest in anthocyanins by contribution to other extracts $e . g$., aqueous and ether extracts.
Analytical results obtained previously, we opted for the purification of two fractions butanol and ethyl acetate extract of acetone-water (L. feei).

The spectroscopic analysis was performed on 11 compounds isolated from the acetone-water extract distributed in the three organs as follows: 6 from twigs, 4 from stems and 1 from leaves. The spectroscopic analysis (UV, IR and NMR) shows that six compounds isolated from twigs fraction were identified as flavones structures (1a-6a) $)^{12,13}$, four products isolated from stems fraction identified as isoflavone structures (7b-10b) $)^{14,15}$, and one compound isolated from leaves extract attributed to the tannin class (11c).

Pentamethoxy-6,7,8,3',4'-hydroxy-5 flavone (1a): $\mathrm{UV}(\mathrm{MeOH})$ : maxima at 281 and $342 \mathrm{~nm}$, ESI-Ms: $m / z\left[\mathrm{MH}^{+}\right]$: 389. NMR H: 12.70 (s,1H), 7.63 (dd, 9Hz, 2Hz, 1H), 7,50 (d, 2Hz, 1H), 7, 09 (d, 9Hz, 1H), 6,99 (s, 1H), 4.02 (s, 3H), 3.90 $(\mathrm{S}, 3 \mathrm{H}), 3.86(\mathrm{~S}, 3 \mathrm{H}), 3.84(\mathrm{~s}, 3 \mathrm{H}), 3,81(\mathrm{~s}, 3 \mathrm{H}) \mathrm{NMR}{ }^{13} \mathrm{C}$ : 163.50 (C2), 103.45 (C3), 182.1 (C4), 148.49 (C5), 135.70 (C6), 152.40 (C7), 132.51 (C8), 145.00 (C9), 106.10 (C10), $122,60\left(\mathrm{C}^{\prime}\right), 111.70\left(\mathrm{C} 2^{\prime}\right), 149.00\left(\mathrm{C} 3^{\prime}\right), 152.30\left(\mathrm{C}^{\prime}\right), 109.05$ (C5'), 119.90 (C6'), 60.50 (6-OMe), 61.70 (7-OMe), 61.50 (8-OMe), 55.71 (3'-OMe), 55.65 (4'-OMe).

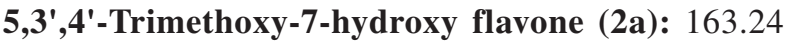
(C-2), 103.77 (C-3), 181.75 (C-4), 151.02 (C-5), 98.78 (C-6), 164.15 (C-7), 132.90 (C-8), 146.63 (C-9), 116.07 (C-10), 122.82 (C-1'), 109.35 (C-2'), 148.93 (C-3'), 152.05 (C-4'), 111.60 (C-5'), 119.96 (C-6'), 55.60 (3'-OMe), 55.54 (4'-OMe).

TABLE-1

EXTRACTION AND TLC ANALYSIS OF LEAVES, STEM AND TWIG FRACTIONS OF WATER-ACETONE EXTRACT (E1: ACETONE /TOLUENE/AC FORMIC 6/8/1, E2: ETHYL ACETATE/MeOH/WATER (10/1.5/1))

\begin{tabular}{|c|c|c|c|c|c|c|}
\hline \multicolumn{7}{|c|}{ Leaves extract } \\
\hline Fraction & Yield $(\%)$ & Colour fraction & $\mathrm{R}_{\mathrm{f}}(\mathrm{E} 1)$ & $\mathrm{R}_{\mathrm{f}}(\mathrm{E} 2)$ & Colour flurescent & $\lambda(\mathrm{nm}) \mathrm{UV}$ \\
\hline Ethyl ether & 0.30 & Yellow & $\begin{array}{l}0.30 \\
0.49 \\
0.57 \\
0.69\end{array}$ & $\begin{array}{l}0.70 \\
0.80 \\
0.90\end{array}$ & Purple & 254 \\
\hline Ethyl acetate & 0.62 & Yellow enib & $\begin{array}{l}0.06 \\
0.13 \\
0.20 \\
0.27 \\
0.46\end{array}$ & $\begin{array}{l}0.55 \\
0.60 \\
0.80\end{array}$ & Purple & 254 \\
\hline Butanol & 0.81 & Red brick & - & 0.85 & Purple & 254 \\
\hline \multicolumn{7}{|c|}{ Stems extract } \\
\hline Ethyl ether & 0.15 & Pale yellow & $\begin{array}{l}0.53 \\
0.66 \\
0.77\end{array}$ & - & Violet & 365 \\
\hline Butanol & 1.51 & Lemon yellow & 0.57 & - & Violet & 365 \\
\hline \multicolumn{7}{|c|}{ Twigs extract } \\
\hline Ethyl ether & 0.37 & Pale yellow & $\begin{array}{l}0.30 \\
0.50 \\
0.60 \\
0.66\end{array}$ & - & Violet & 365 \\
\hline Ethyl acetate & 0.48 & Yellow & $\begin{array}{l}0.36 \\
0.50 \\
0.60 \\
0.66\end{array}$ & - & Purple & $\begin{array}{l}254 \\
365\end{array}$ \\
\hline Butanol & 0.34 & Maron & $\begin{array}{l}0.30 \\
0.50 \\
\end{array}$ & - & Purple & 365 \\
\hline
\end{tabular}


<smiles>[R6]c1cc(-c2oc3c([R])c([R2])c([R9])c([R])c3c(=O)c2[R8])cc([R6])c1[R6]</smiles>

a

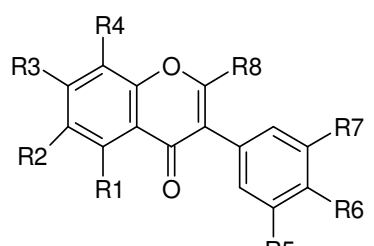

b<smiles>O=C(OC1Cc2c(O)cc(O)cc2OC1c1cc(O)c(O)c(O)c1)c1cc(O)c(O)c(O)c1</smiles>

$11 \mathrm{c}$

$\begin{array}{ccccccccc} & \text { R1 } & \text { R2 } & \text { R3 } & \text { R4 } & \text { R5 } & \text { R6 } & \text { R7 } & \text { R8 } \\ \text { 1a } & \text { OH } & \text { OMe } & \text { OMe } & \text { OMe } & \text { OMe } & \text { OMe } & \text { H } & \text { H } \\ \text { 2a } & \text { OMe } & \text { H } & \text { OH } & \text { H } & \text { OMe } & \text { OMe } & \text { H } & \text { H } \\ \text { 3a } & \text { OH } & \text { H } & \text { OH } & \text { OH } & \text { OMe } & \text { OMe } & \text { H } & \text { H } \\ \text { 4a } & \text { OH } & \text { OH } & \text { OH } & \text { H } & \text { OMe } & \text { OMe } & \text { OMe } & \text { H } \\ \text { 5a } & \text { OH } & \text { H } & \text { OMe } & \text { H } & \text { OH } & \text { OMe } & \text { OMe } & \text { OG1 } \\ \text { 6a } & \text { OH } & \text { H } & \text { OMe } & \text { H } & \text { OH } & \text { OMe } & \text { OMe } & \text { OH } \\ \text { 7b } & \text { OH } & \text { OMe } & \text { OMe } & \text { OMe } & \text { OMe } & \text { OMe } & \text { H } & \text { H } \\ \text { 8b } & \text { OH } & \text { H } & \text { OH } & \text { H } & \text { OMe } & \text { OMe } & \text { H } & \text { H } \\ \text { 9b } & \text { H } & \text { H } & \text { OMe } & \text { G2 } & \text { H } & \text { OMe } & \text { H } & \text { H } \\ \text { 10b } & \text { H } & \text { H } & \text { OH } & \text { G3 } & \text { H } & \text { OMe } & \text { H } & \text { H }\end{array}$

G1= Glucoside-6"-malonyl, 2"-ramnosyl

5,7,8-Trihydroxy-3',4'-dimethoxy flavone (3a): 161.84 (C-2), 104.89 (C-3), 176.78 (C-4), 161.35 (C-5), 113.84 (C-6), 150,47 (C-7), 132.90 (C-8), 146.63 (C-9), 116.86 (C-10), 123.77 (C-1'), 109.59 (C-2'), 148.92 (C-3'), 151.69 (C-4'), 111.67 (C-5'), 119.90 (C-6'), 55.67 (3'-OMe), 55.61 (4'-OMe).

5,6,7-Trihydroxy-3',4'-5-trimethoxy flavone (4a): UV $(\mathrm{MeOH})$ : maxima at 288 and $329 \mathrm{~nm} ;{ }^{13} \mathrm{C}$ NMR: 163.0 (C2), 102.8 (C3), 181.9 (C4), 147.0 (C5), 129.1 (C6), 153.3 (C7), 93.8 (C8), 149.6 (C9), 104.0 (C10), 122,70 (C1'), 111.73 (C2'), 149.00 (C3'), 152.20 (C4'), 109.0 (C5'), 119.90 (C6'), 55.70 (3'-OMe), 55.60 (4'-OMe), 55.71 (5'-OMe).

3-O-(Glucoside-6"'-malonyl, 2"'-ramnosyl)-7,4',5'trimethoxy-5,3'-dihydroxy flavonol (5a): UV(MeOH): 281 , 296, 334, IR (KBr, $\left.v_{\max }, \mathrm{cm}^{-1}\right): 3393(\mathrm{OH}), 2951,2923,2862$ $\left(\mathrm{CH}_{3}, \mathrm{CH}_{2}\right), 1711\left(\mathrm{CH}_{3} \mathrm{COO}\right), 1640(\mathrm{C}=\mathrm{O}, \mathrm{C}-4), 1601(\mathrm{C}=\mathrm{C})$ $1514\left(\mathrm{C}=\mathrm{C}\right.$ arom), 1028, 1121 (C-O) ${ }^{1} \mathrm{H}$ NMR: 6.70 (H-6), 6.84 (H-8), 7.20 (H-2'), 6.93 (H-6'), 3.117, 3.057, 3.229 (O$\left.\mathrm{CH}_{3}\right)$ 3-glycosyl: 5.55 (H-1), $3.697(\mathrm{H}-2), 3.57(\mathrm{H}-3), 3.397$ (H-4), 3.292 (H-5), 3.801 (H-Ga), 3.397 (H-G-b). 2"-ramnosyl: 5.21 (H-1), 4.702 (H-2), 3.729 (H-3) 3.397 (H-4), 4.1 (H-5), $1.03\left(\mathrm{H}-6, \mathrm{CH}_{3}\right), 1.912,1.988,2.086,2.177,2.235,2.027$ $\left(\mathrm{CH}_{3} \mathrm{COO}\right) .{ }^{13} \mathrm{C}$ NMR: 162.24 (C-2), 129.9 (C-3), 174.06 (C4), 151.67 (C-5), 129.9 (C-6), 140.1 (C-7), 99.79 (C-8), 158.44 (C-9), 105.91 (C-10), 122.53 (C-1'), 109.14 (C-2'), 140.1 (C3'), 145.81 (C-4'), 137.81 (C-5'), 109.14 (C-6'), 47.77, 48.11,
48.44, $48.78\left(\mathrm{CH}_{3}-\mathrm{O}\right), 3$-glucosyl : 100.37 (C-1), 79.17 (C-2), 77.23 (C-3), 71.49 (C-4), 73.65 (C-5), 63.37 (C-6) 2"ramnosyl: $102.62(\mathrm{C}-1), 72.68(\mathrm{C}-2), 72.35$ (C-3), 75.07 (C4), 70.43 (C-5), 18.3 (C-6), 6"-malonyl: $41.4\left(\mathrm{CH}_{2}\right), 28.99$, 30.07, 34.9, 38.68, $\left(\mathrm{CH}_{3} \mathrm{COO}\right)$.

5,3'-Dihydroxy-7,4',5'-trimethoxy flavonol (6a): UV $(\mathrm{MeOH})$ : maxima a 286 and $312 \mathrm{~nm}$, IR $\left(\mathrm{KBr}, \mathrm{v}_{\max }, \mathrm{cm}^{-1}\right)$ : 3410, 2934, 2847, 1689, 1558, 1430, 1377, 1115, 1033, 771. ${ }^{1} \mathrm{H}$ NMR: 6.75 (H-6), 6.89 (H-8), 7.19 (H-2'), 6.93 (H-6'), 3.12, 3.06, $3.23\left(\mathrm{O}_{-} \mathrm{CH}_{3}\right),{ }^{13} \mathrm{C}$ NMR: $162.16(\mathrm{C}-2), 130.0(\mathrm{C}-3)$, 174.20 (C-4), 151.67 (C-5), 130.0 (C-6), 140.1 (C-7), 99.78 (C-8), 158.44 (C-9), 105.91 (C-10), 122.53 (C-1'), 109.0 (C2'),140.1 (C-3'), 145.81 (C-4'), 137.81 (C-5'), 109.0 (C-6'), 56.16 (7-OMe), 55.68 (5'-OMe), 55.69 (4'-OMe).

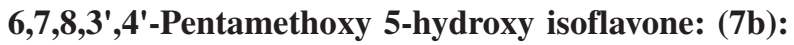
${ }^{1} \mathrm{H}$ NMR: 8.03 (H-2, s), 7.11 (H-2', d, 1.51), 6.98 (H-5', d, 8.4), 6.97 (H-6', dd, 1.51, 8.4), 3.81 (4'-Me, s), 3.78 (3'-Me, s), ${ }^{13} \mathrm{C}$ NMR: 154.55 (C-2), 126.0 (C-3), 181.9 (C-4), 164.51 (C-5), 103.30 (C-6) 161.62 (C-7), 99.28 (C-8), 159.40 (C-9), 108.0 (C-10), 124.91 (C-1'), 117.33 (C-2'), 147.50 (C-3'), 149.40 (C-4'), 60.58 (6-OMe), 61.79 (7-OMe), 61.49 (8-OMe), 56.43 (4'-OMe), 55.67 (3'-OMe).

5,7-Dihydroxy 3',4'-dimethoxy isoflavone (8b): ${ }^{1} \mathrm{H}$ NMR: 8.12 (S, H-2), 6.57 (H-6, d, 2.0), 6.73 (H-8, d, 2.0), 7.06 (H-2', d, 1.50), 6.98 (H-5', d, 8.5), 6.9è (H-6', dd, 1.5, 8.5), 3.87 (4'$\mathrm{Me}, \mathrm{s}), 3.81$ (3'-Me, s), ${ }^{13} \mathrm{C}$ NMR: 155.55 (C-2), 125.01 (C-3), 182.3 (C-4), 164.73 (C-5), 101.30 (C-6) 163.62 (C-7), 99.98 (C-8), 159.30 (C-9), 108.10 (C-10), 124.94 (C-1'), 117.31(C2'), 147.54 (C-3'), 149.40 (C-4'), 56.48 (4'-OMe), 55.68 (3'-OMe).

Hexacetate 8-hydroxy-4',7-dimethoxy isoflavone 8-O[ $\alpha$-rhamnopyranosyl-(1-6)]- $\beta$-glucopyranoside (9b): $\mathrm{C}_{41} \mathrm{H}_{47} \mathrm{O}_{20},[\mathrm{M}+\mathrm{H}]^{+}$a $m / z=859, \mathrm{UV}(\mathrm{MeOH}): 212,253,306$ nm. IR (KBr, $\left.v_{\max }, \mathrm{cm}^{-1}\right)$ : 3404, 3235, 2918, 2841, 1629, 1514 , 1377, 1115, 1039. ${ }^{1} \mathrm{H}$ NMR: 8.25 (s, H-2), 8.00 (d, 9.0, H-5), 7.50 (d, 8.5, H-2', H-6'), 7.27 (d, 9.0, H-6), 6.99 (d, 8.5, H-3', H-5'), 5.07 (d, 7.5, H-1"), 4.56 (d, 1.5, H-1'"), 4.03 (s, OMe), 3.82 (s, OMe) $3.84-3.37$ (m, glucose and rhamnose protons), 1.06 (d, 6.5, H-6"'). ${ }^{13} \mathrm{C}$ NMR: 152.7 (C-2), 124.7 (C-3), 176.0 (C-4), 119.4 (C-10), 123.4 (C-5), 110.3 (C-6), 156.1 (C-7), 132.4 (C-8), 150.8 (C-9), 124.2 (C-1'), 130.5 (C-2', C-6'), 114.2 (C-3', C-5'), 159.9 (C-4'), 101.2 (C-1"), 72.1 (C-2"), 72.9 (C-3") 69.2 (C-4"), 74.4 (C-5"), 66.5 (C-6"), 97.9 (C-1"'), 69.6 (C-2"'), 69.3 (C-3"'), 70.9 (C-4"'), 66.9 (C-5"'), 17.5 (C-6"'), 56.8 (7-OMe), 55.6 (4'-OMe), 21.0, 20.9, 20.9, 20.8 ( $\left.\mathrm{CH}_{3} \mathrm{CO}\right)$. $170.5,170.3,170.2,170.1,19.9,169.6\left(\mathrm{COCH}_{3}\right)$.

Tetraacetate 7,8-dihydroxy-4'-methoxyisoflavone 8-OB-glucopyranoside (10b): UV(MeOH): 211, 254, 306, IR $\left(\mathrm{KBr}, \mathrm{v}_{\max }, \mathrm{cm}^{-1}\right): 3448,2918,2852,1727,1590,1519,1170$, 1022, 1465, 716. ${ }^{1} \mathrm{H}$ NMR: 7.85 (s, H-2), 8.05 (d, 9.0, H-5), 7.05 (d, 9.0, H-6), 7.47 (d, 9.0, H-2', H-6'), 6.98 (d, 9.0, H-3', H-5'), 4.99 (d, 8.1, H-1"), 5.4 (dd, 9.8, 8.1, H-2"), 5.33 (t, 9.8, H-3"), 5.2 (t, 9.8, H-4"), 3.82 (ddd, 9.8, 5.6, 2.8, H-5"), 4.3 (dd, 12.6, 5.6, H-6"), 3.84 (s, 4'-OMe), 2.20, 2.12, 2.07, 2.06 $\left(\mathrm{CH}_{3} \mathrm{CO}\right) .{ }^{13} \mathrm{C}$ NMR: $151.3(\mathrm{C}-2), 125.1(\mathrm{C}-3), 175.6(\mathrm{C}-4)$, 118.7 (C-10), 124.3 (C-5), 115.3 (C-6), 154.4 (C-7), 131.1 (C-8), 150.2 (C-9), 123.5 (C-1'), 130.2 (C-2', C-6'), 114.1 (C3', C-5'), 159.9 (C-4'), 103.4 (C-1"), 71.0 (C-2"), 72.1 (C-3") 67.9 (C-4"), 72.7 (C-5"), 61.3 (C-6"), 55.4 (4'-OMe), 20.6, $20.7\left(\mathrm{CH}_{3} \mathrm{CO}\right), 17.6,170.1,169.3,169.2\left(\mathrm{COCH}_{3}\right)$. 


\section{ACKNOWLEDGEMENTS}

The authors are gratful to MESRS and DGRST for the financial support given through this project.

\section{REFERENCES}

1. P. Quezel and S. Santa, Nouvelle Flore de l'Algérie et des régions désertiques et Meridionales, Tome II, édition CNRS, Paris (1963).

2. P. Ozenda, Flore du Sahara. Ed. CNRS: Paris, (1983).

3. A. Cheriti, Plantes médicinales de la région de Bechar, sud ouest Algérie: Etude Ethnopharmacologique. Rapport CRSTRA, Algerie, (2000)

4. N. Belboukhari and A. Cheriti, Asian J. Plant Sci., 4, 496 (2005).

5. N. Belboukhari and A. Cheriti, Res. J. Phytochemistry, 1, 74 (2007)

6. A. Cheriti, N. Belboukhari and S. Hacini, Iran. J. Pharm. Res., 3, 51 (2004).
7. E. Camacho-Frias and A.P. Foucault, Analusis, 24, 159 (1996).

8. K. Hostettmann, B. Domon, D. Schaufelberger and M. Hostettmann, J. Chromatogr. A, 283, 137 (1984).

9. J.B. Harborne, (1986). The Flavonoids. Chapman \& Hall, London.

10. M.O. Hamburger and G.A. Cordell, J. Nat. Prod., 50, 19 (1987).

11. K. Hostettman, A. Marston and M. Hostettman, (1998), preparative chromatography techniques. Applications in natural product isolation, $2^{\text {nd }}$ edt, Springer, Berlin Heidelberg.

12. J.D. Bacon and T.J. Mabry, Rev. Latinoam. Quim., 7, 83 (1976).

13. T.J. Mabry, K.R. Markham and H.B. Thomas, The systematic identification of flavonoids. Springer Verlag, Heidelberg, New York (1970).

14. C.C.W. Wanjala and R.R.T. Majinda, Fitoterapia, 71, 400 (2000)).

15. K.R. Markham and H. Geiger, in ed: J.B. Harborne, ${ }^{1} \mathrm{H}$ NMR Spectroscopy of Flavonoids, Advances in Research Since 1986, pp.1-676, Chapman \& Hall, London (1994) 